\title{
Development and application of a set of mesh-based and age- dependent Chinese family phantoms for radiation protection dosimetry: Preliminary Data for external photon beams
}

\author{
Yifei $\mathrm{Pi}^{1}$, Lian Zhang ${ }^{1}$, Wanli Huo ${ }^{1}$, Mang Feng ${ }^{1}$, Zhi Chen ${ }^{1}$ and X. George $\mathrm{Xu}^{1,2, *}$ \\ ${ }^{1}$ University of Science and Technology of China, Hefei, China \\ ${ }^{2}$ Rensselaer Polytechnic Institute, Troy, New York, USA
}

\begin{abstract}
A group of mesh-based and age-dependent family phantoms for Chinese populations were developed in this study. We implemented a method for deforming original RPI-AM and RPI-AF models into phantoms of different ages: $5,10,15$ and adult. More than 120 organs for each model were processed to match with the values of the Chinese reference parameters within $0.5 \%$. All of these phantoms were then converted to voxel format for Monte Carlo simulations. Dose coefficients for adult models were counted to compare with those of RPI-AM and RPI-AF. The results show that there are significant differences between absorbed doses of RPI phantoms and these of our adult phantoms at low energies. Comparisons for the dose coefficients among different ages and genders were also made. it was found that teenagers receive more radiation doses than adults under the same irradiation condition. This set of phantoms can be utilized to estimate dosimetry for Chinese population for radiation protection, medical imaging, and radiotherapy.
\end{abstract}

\section{Introduction}

A main and indispensable task in radiation protection, medical imaging, and radiotherapy is to estimate organ doses. Computational phantoms, especially whole body computational phantom for estimations of dosimetry have become widely used $[1,2]$. Such phantoms are typically based on reference anatomical values, for the population of interests. The ICRP firstly used so called "Reference man" to define them in the ICRP Publication 23 in 1975. Since the 1960s, three categories of computational phantoms have appeared, including stylized phantoms, voxel phantoms, and boundary representation (BREP) phantoms [1]. Compared with stylized phantoms, voxel phantoms utilize huge building blocks and mix them together. Each block has their own density data and material information based on medical images such as CT or MRI. Further, variability in shape and mess define introduced by motion of human body can be a significant factor affecting organ dose. However, changing phantoms in the voxel format is extremely inefficient. BREP phantoms, in the form of either Non-Uniform Rational BSpline (NURBS) or polygonal meshes, possess attractive features in flexibility and anatomical realism $[3,4,5]$. China is rapidly developing its nuclear power industry and its radiation protection system follows those recommended by the ICRP. However, the ICRP Computational Phantoms are based on western populations and are not representative of the Chinese. Most reported Chinese phantoms are designed for Chinese adults (CNMAN 、 $\mathrm{VCH}$ and CVP) $[6,7,8]$, there are currently no phantoms for Chinese minors. This paper reports our preliminary efforts in developing a set of mesh-based and agedependent Chinese family phantoms for the purposes of deriving radiation protection dosimetry data.

\section{Materials and methods}

Figure 1 illustrates steps of the developing process. The process starts with the collection of initial input data including human dimensions of Chinese adults and minors $[9,10]$, reference organ volume information and the RPIAM and RPI-AF mesh phantoms. The deforming process was divided into two parts: human dimensions scaling and organ volume deformation. The first part was finished by some scripts in Matlab form automatically and the organ volume deformation step was done manually through Rhinoceros, a CAD software. We utilized an in-house C\# based voxelization tool [11] to convert the meshed-based age-dependent phantoms into voxel formatted phantoms since polygonal mesh cannot be able to handle straight forward by MCNPX [12]. The organ doses and effective doses of our USTC adult phantoms are compared with those from RPI-AM and RPI-AF with six classic source directions (AP, PA, LLAT, RLAT, ROT, ISO) at energies from $20 \mathrm{keV}$ to $10 \mathrm{MeV}$ [13]. This paper also reports our attempts on comparisons of the dose coefficient for phantoms with different ages and genders.

\footnotetext{
* Corresponding author: xgxu@ustc.edu.cn
} 


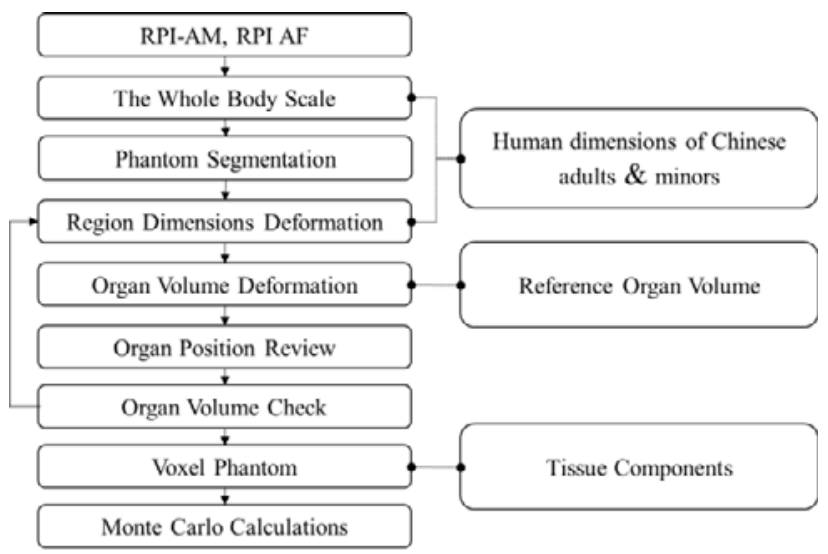

Figure 1. Flowchart of construction of mesh-based and agedependent family phantoms

\subsection{Original models and reference parameters}

RPI-AM and RPI-AF, which were developed at RPI, are a pair of adult male and adult female computational phantoms and are compatible with anatomical parameters for the 50th percentile population as specified by the ICRP publication 89 [14]. Up to 140 inner organs or tissues from original 500 available structures were filtrated and designed to represent the whole human body. The organ volume were automatically adjusted to match the reference value. And they were all designed entirely with polygonal mesh surface that affords the ability to sufficiently deform the dimensions of phantoms and the shape of organs. The pair of phantoms are fully validated for their accuracy [4]. Furthermore, researchers in RPI have resoundingly extended the original reference phantoms to represent different populations and obese patients $[11,15]$. For all these reasons, we selected RPI-AM and RPI-AF as our initial phantoms.

Corresponding to ICRP reports, Chinese government issued reference individuals for radiation protection, a national standard to standardize Chinese "Reference Man" [10], whose organ size and mass information are contained. Some important data are listed in Table 1. However, we could not find all data for every organ or tissue in published Chinsed standards. Organ contents and some radial insensitive tissue, such as muscles, vessel, are missed. We took notice of that the IAEA (International atomic energy agency) group published some anatomical, physiological and metabolic characteristics for "Reference Asian Man" with the age group as new-born, 1 year, 5 years, 10 years and adult from 20 to 50 as the ICRP done for white people $[16,17,18]$. Apparently, we could adopt these data for Chinese people as a member of them. Particularly, we made use of the ICRP proportion data of the bones to calculate the mass of the red bone marrow (RBM), yellow bone marrow (YBM), trabecular and cortical part for every part of phantom bones [19,20]. It was because that we cannot find enough useful information from Chinese standards and Asian reference data.
Table 1. Reference values (g) for masses of important organs/tissue for Chinese

\begin{tabular}{ccccccc}
\hline \multirow{2}{*}{$\begin{array}{c}\text { organs/ } \\
\text { tissue }\end{array}$} & \multirow{2}{*}{$\mathbf{5}$} & \multirow{2}{*}{$\mathbf{1 0}$} & \multicolumn{2}{c}{$\mathbf{1 5}$ years } & \multicolumn{2}{c}{$\mathbf{2 0 - 5 0}$ years } \\
\cline { 5 - 7 } & years & years & male & female & male & female \\
\hline Testicles(2) & 3.1 & 4.7 & 33 & - & 40 & - \\
Ovaries(2) & 0.5 & 1.4 & - & 9.8 & - & 11 \\
RBM & 305 & 710 & 900 & 750 & 1100 & 800 \\
Colon & 102 & 170 & 291 & 225 & 310 & 240 \\
Lungs & 360 & 580 & 940 & 720 & 1250 & 960 \\
Stomach & 47 & 75 & 120 & 95 & 145 & 110 \\
Urinary & 13 & 21 & 38 & 30 & 40 & 30 \\
bladder & & & & & & \\
Mammary & - & 38 & - & 200 & - & 300 \\
gland & & & & & & \\
Liver & 575 & 850 & 1170 & 1050 & 1410 & 1290 \\
Esophagus & 13 & 25 & 30 & 28 & 40 & 30 \\
Thyroid & 3.4 & 7.9 & 12 & 12 & 20 & 17 \\
Skin & 775 & 1200 & 2200 & 1700 & 2400 & 1800 \\
Bone & & & & - & 100 & 90 \\
surface & - & - & - & - &
\end{tabular}

Table 2. Reference values ( $g$ ) for masses of other organs/tissue for Chinese

\begin{tabular}{ccccccc}
\hline \multirow{2}{*}{$\begin{array}{c}\text { organs/ } \\
\text { tissue }\end{array}$} & $\mathbf{5}$ & $\mathbf{1 0}$ & \multicolumn{2}{c}{$\mathbf{1 5}$ years } & \multicolumn{2}{c}{$\mathbf{2 0 - 5 0}$ years } \\
\cline { 6 - 7 } years & years & male & female & male & female \\
\hline Body fat & 1900 & 5000 & 6500 & 9000 & 9000 & 12500 \\
LBW & 17100 & 27000 & 48500 & 41000 & 54000 & 41500 \\
Skeleton & 2200 & 4500 & 7300 & 5700 & 8000 & 6000 \\
Brain & 1200 & 1350 & 1480 & 1360 & 1460 & 1330 \\
Heart & 95 & 150 & 240 & 200 & 325 & 290 \\
Kidney(2) & 115 & 175 & 230 & 220 & 290 & 260 \\
Spleen & 70 & 100 & 140 & 120 & 165 & 150 \\
Sialaden & 26 & 45 & 77 & 59 & 82 & 62 \\
$\begin{array}{c}\text { Gall } \\
\text { bladder }\end{array}$ & 3 & 4 & 8 & 6 & 9 & 7 \\
$\begin{array}{c}\text { Small } \\
\text { intestine }\end{array}$ & 190 & 325 & 540 & 420 & 620 & 450 \\
Pancreas & 40 & 60 & 90 & 75 & 120 & 100 \\
$\begin{array}{c}\text { Eyes } \\
\text { Eye lens }\end{array}$ & 13 & 14 & 15 & 12 & 15 & 12 \\
$\begin{array}{c}\text { Adrenal } \\
\text { gland }\end{array}$ & 0.35 & 0.35 & 0.4 & 0.35 & 0.4 & 0.35 \\
$\begin{array}{c}\text { Thymus } \\
\text { Pituitary }\end{array}$ & 33 & 6 & 10 & 10 & 14 & 13 \\
gland & 0.3 & 0.4 & 0.53 & 0.61 & 0.7 & 0.8 \\
$\begin{array}{c}\text { Body } \\
\text { weight }\end{array}$ & 19000 & 32000 & 55000 & 50000 & 63000 & 54000 \\
\hline
\end{tabular}


Table 3. Reference values (g) for masses of organs/tissue for Asian Reference Man

\begin{tabular}{|c|c|c|c|c|c|c|c|c|}
\hline \multirow{2}{*}{$\begin{array}{l}\text { organs/ } \\
\text { tissue }\end{array}$} & 5 & 5 & 10 & 10 & 15 & 15 & Adult & Adult \\
\hline & M & $\mathrm{F}$ & M & $\mathrm{F}$ & $\mathrm{M}$ & $\mathrm{F}$ & M & $\mathrm{F}$ \\
\hline $\begin{array}{c}\text { Colon } \\
\text { contents }\end{array}$ & 120 & 120 & 190 & 190 & 340 & 260 & 360 & 280 \\
\hline $\begin{array}{l}\text { Stomach } \\
\text { contents }\end{array}$ & 80 & 80 & 130 & 130 & 230 & 170 & 240 & 180 \\
\hline $\begin{array}{l}\text { Bladder } \\
\text { contents }\end{array}$ & 33 & 32 & 54 & 54 & 96 & 78 & 100 & 85 \\
\hline $\begin{array}{c}\text { Heart } \\
\text { contents }\end{array}$ & 130 & 130 & 210 & 220 & 380 & 290 & 400 & 300 \\
\hline $\begin{array}{l}\text { Gall bladder } \\
\text { contents }\end{array}$ & 16 & 16 & 27 & 29 & 47 & 36 & 50 & 38 \\
\hline $\begin{array}{l}\text { Small intestine } \\
\text { contents }\end{array}$ & 190 & 190 & 190 & 190 & 330 & 260 & 350 & 270 \\
\hline Rectum & 6 & 6 & 10 & 10 & 19 & 19 & 20 & 20 \\
\hline Prostate & 1 & - & 1.5 & - & 11 & - & 12 & - \\
\hline $\begin{array}{l}\text { Prostate } \\
\text { contents }\end{array}$ & 0.34 & - & 0.51 & - & 3.6 & - & 4 & - \\
\hline Tongue & 64 & 50 & 67 & 51 & 67 & 51 & 67 & 51 \\
\hline Tonsil & 1 & 0.98 & 1 & 1 & 4 & 3 & 4 & 3 \\
\hline Bronchus & 8.5 & 8.3 & 14 & 15 & 24 & 18 & 26 & 20 \\
\hline Trachea & 3.2 & 2.9 & 5 & 5 & 8 & 6 & 9 & 6.8 \\
\hline Ureter & 5.1 & 4.9 & 8 & 8.6 & 13 & 14 & 14 & 15 \\
\hline Uterus & - & 5.9 & - & 8.9 & - & 63 & - & 70 \\
\hline Spinal cord & 20 & 20 & 30 & 30 & 30 & 30 & 30 & 30 \\
\hline Teeth & 45 & 34 & 45 & 34 & 45 & 34 & 43 & 34 \\
\hline
\end{tabular}

\subsection{Phantom adjustment}

To obtain a phantom with a given height, we developed a uniform scaling factor adjustment method for adult phantoms and different factors for different parts in teenagers' deformation. The growth rate is fairly slow after 18 years old for boys and 16 years old for girls. It is reasonable to deform phantoms among adults using uniform scaling [15,21]. But for adolescents, growth and development occurs more rapidly for arms, legs and the trunk. For that reason, we split the original models into 4 parts, the head part, the arms part, the trunk part and the leg part. Figure 2 illustrates our distributing standard. As is shown in the picture, the head part are those structures from top of the model to gnathion and organs or tissue in the section from gnathion to perineal constitute the trunk part. According to the length, width and heightof every part, we applied the uniform scaling factor adjustment method. Scale factors were calculated by using:

$$
\begin{aligned}
& \text { WFactor }=\text { AIMWidth } / \text { Width } \\
& \text { HFactor }=\text { AIMHeight } / \text { Height } \\
& \text { LFactor }=\text { AIMLength } / \text { Length }
\end{aligned}
$$

PointNew $=($ Point. $x *$ WFactor, Point. $y *$ HFactor, Point. $Z *$ LFactor) where AIMWidth is the reference width of our model part, Width represents the actual width value. AIMHeight, Height, AIMLength and Length have similar significances. Point.x, Point.y and Point.z are coordinates of vectors on our original models. PointNew is the newly calculated point.

After this step, we assembled all the parts together to build one whole body model. Some reparative attempts were finally took in order to smooth discrete commissures. All of these steps for scaling were completed automatically with some in-house MATLAB R2014B[22] scripts except for the reparative works. We finished human dimension adjustment automatically and obtained rough phantoms.

However, those elementary models could not be utilized because of their rough anatomic details. In order to match internal organ volume with the Chinese reference parameters, a 3D model software named Rhinoceros 5 was used. By carrying out mesh offset, mesh scale and box edit operations, we can change organs' volume and shape. Figure 3 displays the deformation methods. We manually took efforts to adjust organs parameters to agree within $0.5 \%$ with the reference people data of China. Finally, all of these regions were assembled together.

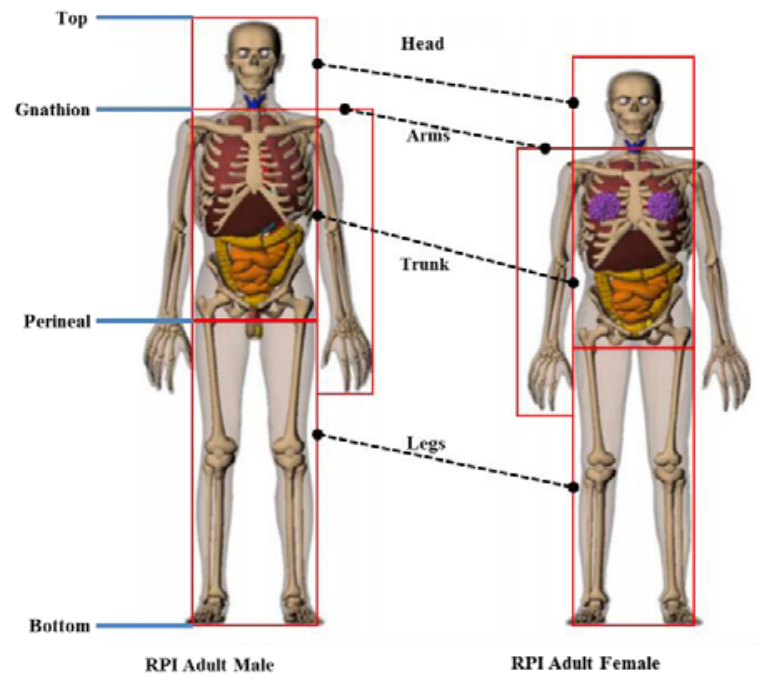

Figure 2. Different scale factors for different parts

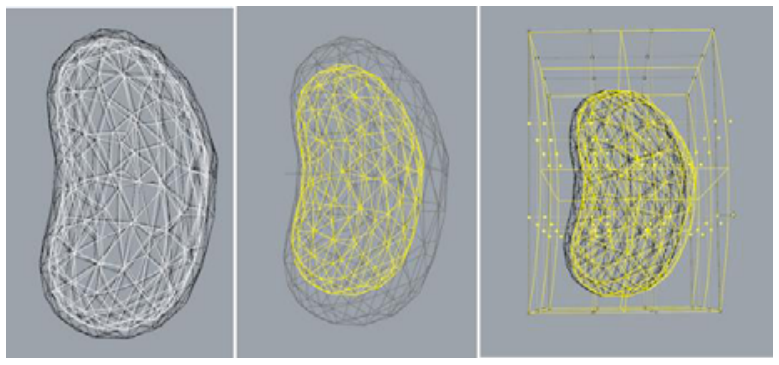

Figure 3. Mesh offset (left), mesh scale (middle) and box edit (right) operations in Rhinoceros

\subsection{Tissue composition and Voxelization}

The tissue element compositions were defined for the purposes of radiation transport simulations involving 
Monte Carlo methods. Organ-specific element compositions were based on reference value of the Chinese standard and the ICRU report $46[23,24]$. A $\mathrm{C}++/ \mathrm{C} \#$ based voxelization tool was used to convert the meshed-based age-dependent phantoms into voxel formatted phantoms. The organ volumes after the voxelization, were found to agree within $1 \%$ with the reference parameters.

\subsection{Dose calculation}

In this project, MCNPX code was used to calculate dose coefficients. We defined "photon and electron"mode in input files for photon simulations. The electron crosssection library el03 and photon cross-section library mcnplib04 were used. Monoenergetic and parallel photon beams from 6 normal source geometries: AP, PA, LLAT, RLAT, ROT and ISO, were took into consideration. We simulated incident energies covering 20 values from $20 \mathrm{keV}$ to $10 \mathrm{MeV}$. The deposited energy for each organ was counted using the ${ }^{*} \mathrm{~F} 8$ card. Three correction factor methods, noted by Lee et al [25] and applied by many other works [4,26], were adopted to compute the red bone marrow doses from the bone spongiosa doses. To keep statistical error less than $1 \%$, up to 1 e 8 particles were used for every instance. All simulations were finished on a 24core Linux server with 32GB RAM memory.

The stochastic efforts on the whole body could be evaluated by the effective dose, E, defined by the ICRP [27]. E is computed as weighted organ equivalent doses involving sex-averaged value according to:

$$
E=\sum_{T} \omega_{T}\left[\frac{H_{T}^{M}+H_{T}^{F}}{2}\right]
$$

where $\omega_{T}$ is the weighting factor for specified tissue or organ $T$ defined in ICRP-103, $H_{T}^{M}$ and $H_{T}^{F}$ are the equivalent dose of $T$ in the male and female phantoms, respectively.

\section{Results and discussion}

\subsection{Mesh-based and age-dependent family phantoms}

Eight new mesh-based and age-dependent Chinese phantoms were developed: USTC-AM, USTC-AF, USTC15M, USTC-15F, USTC-10M, USTC-10F, USTC-5M, USTC-5F. A total of 70 internal organs, 45 bone components and 4 muscle structures are contained in each single newly developed phantom. Figure 4 displays 3D rendering pictures of these adult phantoms. Figure 5 represents the geometries for teenager phantoms.

Table 4 and 5 illustrate the dimensions of the meshbased and age-dependent Chinese phantoms. They agree well with the references parameters.

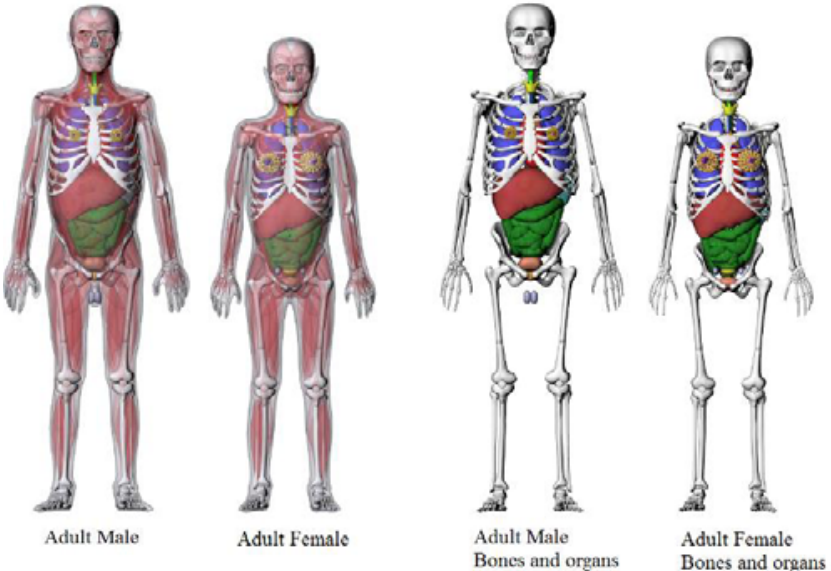

Figure 4. USTC - adult male and female mesh phantoms

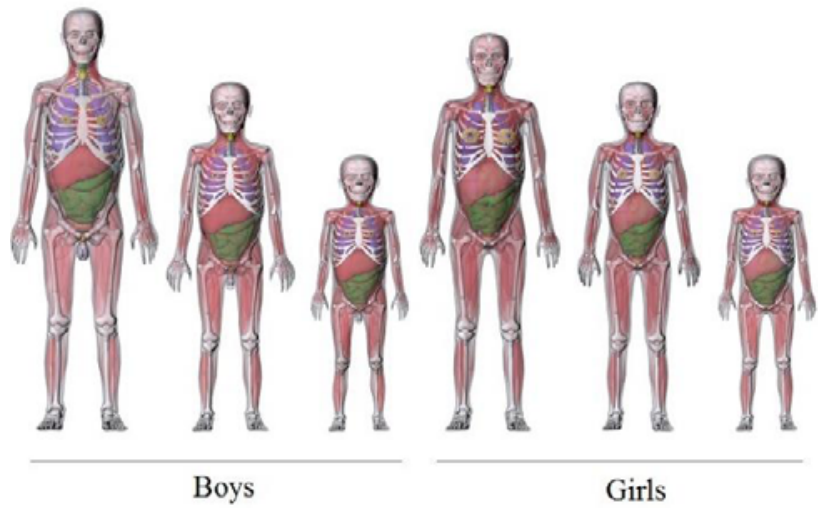

Figure 5. USTC - teenage mesh phantoms

Table 4. The value of stature for USTC phantoms.

\begin{tabular}{cccc}
\hline & Real(cm) & Reference(cm) & $\begin{array}{c}\text { Relative } \\
\text { Error(\%) }\end{array}$ \\
\hline USTC-AM & 170 & 170 & 0 \\
USTC-AF & 158 & 158 & 0 \\
USTC-15M & 168 & 168 & 0 \\
USTC-15F & 158 & 158 & 0 \\
USTC-10M & 139 & 139 & 0 \\
USTC-10F & 139 & 139 & 0 \\
USTC-5M & 110 & 110 & 0 \\
USTC-5F & 110 & 110 & 0 \\
\hline
\end{tabular}

Table 5. The head height for USTC phantoms.

\begin{tabular}{cccc}
\hline Name & Real(cm) & Reference(cm) & $\begin{array}{c}\text { Relative } \\
\text { Error(\%) }\end{array}$ \\
\hline USTC-AM & 23.78 & 23.69 & 0.38 \\
USTC-AF & 22.95 & 22.82 & 0.57 \\
USTC-15M & 23.43 & 23.30 & 0.57 \\
USTC-15F & 22.44 & 22.70 & 1.16 \\
USTC-10M & 22.00 & 21.85 & 0.67 \\
USTC-10F & 21.40 & 21.65 & 1.18 \\
USTC-5M & 20.18 & 20.60 & 2.02 \\
USTC-5F & 20.18 & 20.20 & 0.08 \\
\hline
\end{tabular}

\subsection{Absorbed dose and effective dose of USTC phantoms}

Using standard radiation protection dosimetry methods, monoenergetic and parallel photon beams from AP, PA, 
LLAT, RLAT, ROT and ISO geometries were simulated and calculated doses were normalized by kerma free-in-air. Absorbed dose conversion coefficients for every organ and tissue in USTC phantoms were compared with those from RPI-AM and RPI-AF under the same conditions.

Figure 6 summarizes the relative absorbed doses to the male testicles and figure 7 shows the relative doses to the female ovaries. For low-energy photons, considerable differences are observed between USTC phantoms and RPI phantoms. Because of the anatomical dependency for the relative absorb dose to a particular organ or tissue, these differences are expected. Generally, the dimensions of USTC adult phantoms are less than those of RPI adult phantoms, and the mass value for a single organ is smaller than that in the Caucasian. Therefore, relative absorb doses in USTC adult phantoms are higher. As for high-energy photons, the differences of dose coefficients are relatively small. In the case of the ratio of ovaries absorbed dose, large differences are illustrated in figure 7. USTC-AF and RPI-AF phantoms have the same ovaries' mass, but the ovaries in USTC-AF are further to the posterior side than RPI-AF. And the abdominal width of USTC-AF is smaller than the value of RPI-AF phantom.

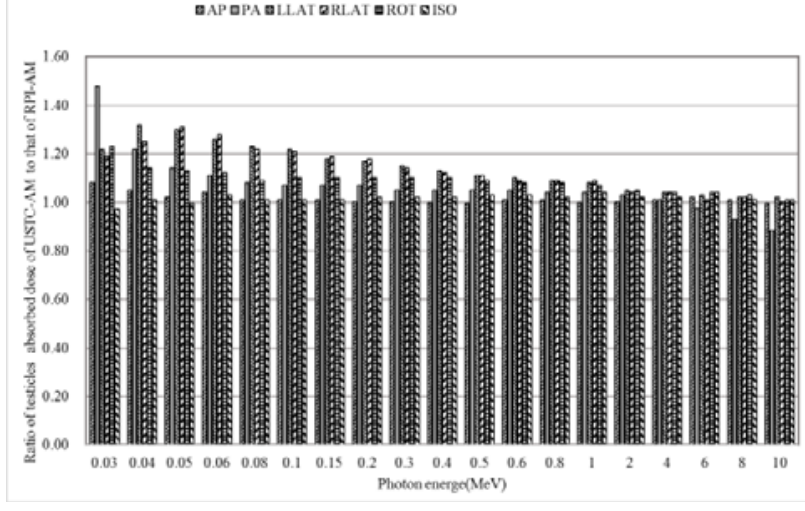

Figure 6. Ratio of testicles absorbed dose for USTC-AM to that of RPI-AM from photon beams.

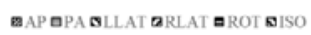

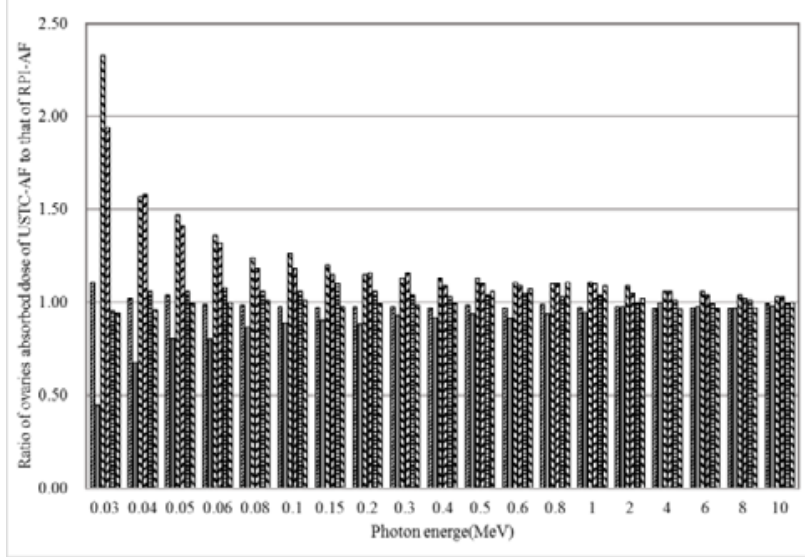

Figure 7. Ratio of ovaries absorbed dose of USTC-AF to that of RPI-AF showing large differences caused by the location of organs and the abdominal width

Figure 8 presents the results of the normalized effective doses for adult, 15-year old, 10-year old and 5-year old phantoms. For each phantom, the AP irradiation geometry shows the largest effective dose. When comparison difference ages, it is found that the 5-year old phantoms have the largest effective dose, due to the fact that the dimensions of smaller phantoms and organs are located closer to the body surfaces.

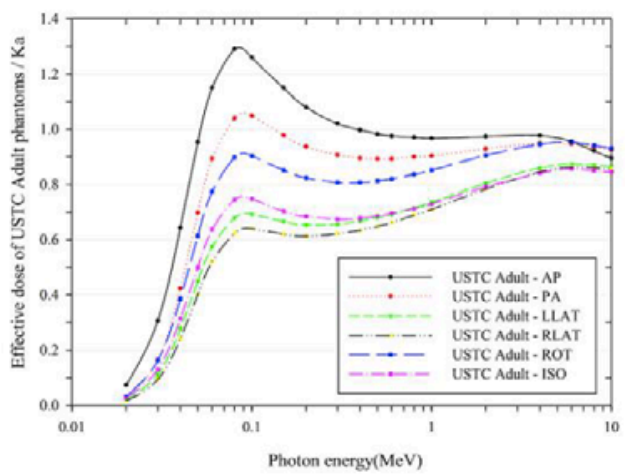

(a)

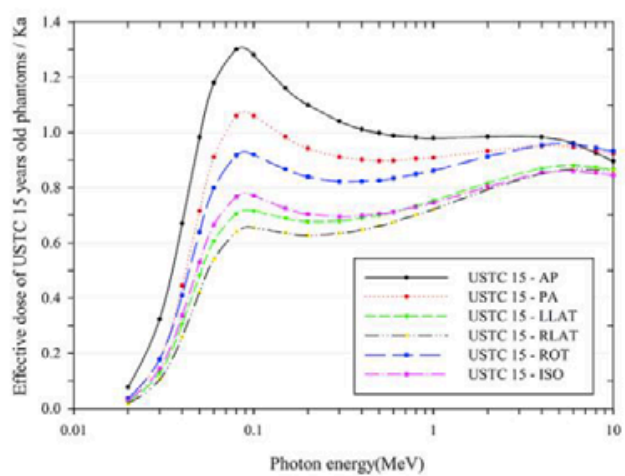

(b)

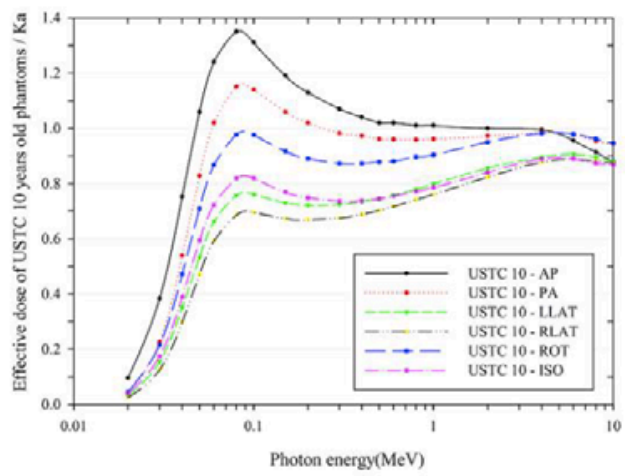

(c)

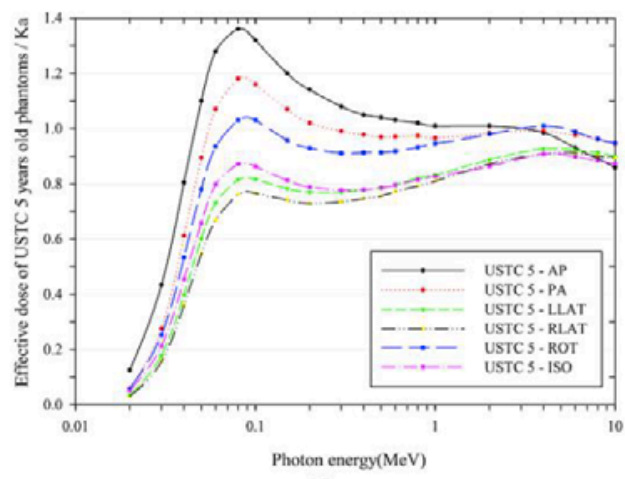

(d)

Figure 8. The normalized effective doses for (a) USTC adult, (b) 15 years old, (c) 10 years old and (d) 5 years old phantoms 


\section{Conclusion}

A set of mesh-based and age-dependent adult and adolescent Chinese phantoms have been developed. Automated and manual methods were implemented to match the phantom parameters with Chinese reference values. All phantoms were voxelized for the purposes of Monte Carlo dose calculations. For low-energy photons, considerable differences are observed between USTC phantoms and those of RPI phantoms, suggesting that it is important to consider population related anatomical information in radiation protection dose calculations.

\section{Acknowledgment}

This work was supported in part by grants from the National Natural Science Foundation of China (Grant No. 11375181).

\section{References}

1. X. G. Xu, Physics in medicine and biology, 59, R233 (2014)

2. X. G. Xu, K. F. Eckerman, Handbook of anatomical models for radiation dosimetry (CRC Press, 2009)

3. L. Su, B. Han, X. G. Xu, Radiation protection dosimetry, ncr194 (2011)

4. J. Zhang, Y. H. Na, P. F. Caracappa, X. G. Xu, Physics in medicine and biology, 54, 5885 (2009)

5. J. Zhang, X. G. Xu, C. Shi, M. Fuss, Journal of applied clinical medical physics/American College of Medical Physics, 9, 2700 (2008)

6. J. Li, R. Qiu, Z. Zhang, L. Liu, Z. Zeng, L. Bi, W. Li, Radiation protection dosimetry, ncp087 (2009)

7. B. Zhang, J. Ma, L. Liu, J. Cheng, Radiation protection dosimetry, 124, 130-136 (2007)

8. G. Zhang, Q. Liu, S. Zeng, Q. Luo, Physics in medicine and biology, 53, 3697 (2008)

9. GB/T 26158-2010 (Chinese Standard Press, Beijing, 2010)

10. GBZ/T200.1-2007 (People's Medical Publishing House, Beijing, 2007)

11. A. Ding, M. M. Mille, T. Liu, P. F. Caracappa, X. G. $\mathrm{Xu}$, Physics in medicine and biology, 57, 2441 (2012)

12. D. B. Pelowitz, Los Alamos National Laboratory, 76, (2005)

13. C. O. R. P. International, ICRP Publication 74: Conversion Coefficients for Use in Radiological Protection Against External Radiation (Elsevier Health Sciences, 1997)

14. J. Valentin, Annals of the ICRP, 32, 1-277 (2002)

15. Y. H. Na, B. Zhang, J. Zhang, P. F. Caracappa, X. G. $\mathrm{Xu}$, Physics in medicine and biology, 55, 3789 (2010)

16. H. KAWAMURA, G. TANAKA, K. SHIRAISHI, Y. HONDA, M. NISHIMUTA, T. KOYANAGI, F. KARIM, M. MOLLA, A. RAB, J. WANG, Journal of radiation research, 39, 350 (1998)

17. G. Tanaka, H. Kawamura, R. V. Griffith, M. Cristy, K. F. Eckerman, Radiation protection dosimetry, 79, 383-
386 (1998)

18. J. X. Wang, R. Chen, H. Zhu, Y. Z. Zhou, R. W. Ma, Data of anatomical physiological and metabolic characteristics for Chinese reference man (Atomic Energy Press, Beijing, 1998)

19. M. Zankl, K. F. Eckerman, W. E. Bolch, Radiation protection dosimetry, 127, 174-186 (2007)

20. C. J. Watchman, D. Hasenauer, W. E. Bolch, Physics in medicine and biology, 52, 3133 (2007)

21. V. F. Cassola, V. J. de Melo Lima, R. Kramer, H. J. Khoury, Physics in medicine and biology, 55, 133 (2009)

22. MathWorks,

23. GBZ/T200.5-2009 (Beijing, People's Medical Publishing, 2009)

24. J. Deasy, Medical Physics, 21, 709-710 (1994)

25. C. Lee, C. Lee, A. P. Shah, W. E. Bolch, Physics in medicine and biology, 51, 5391 (2006)

26. L. Liu, Z. Zeng, J. Li, B. Zhang, R. Qiu, J. Ma, Physics in medicine and biology, 54, 6675 (2009)

27. ICRP, The 2007 Recommendations of the International Commission on Radiological Protection (Elsevier, Oxford, 2007) 